\title{
A High-Performance Optical Time-Domain Brillouin Distributed Fiber Sensor
}

\author{
Silvia Diaz, Stella Foaleng Mafang, Manuel Lopez-Amo, Senior Member, IEEE, and Luc Thévenaz
}

\begin{abstract}
A novel configuration for a Brillouin distributed fiber sensor based on Brillouin optical time-domain analysis is proposed. This configuration eliminates many intensity noise issues found in previous schemes. Resolution of $7 \mathrm{~m}$ all over a $47 \mathrm{~km}$ single-mode fiber was achieved and resolution down to $30 \mathrm{~cm}$ in a few kilometer fiber. Noise reduction makes possible measurements with a 16 times averaging.
\end{abstract}

Index Terms-Brillouin scattering, nonlinear optics, optical fiber sensors.

\section{INTRODUCTION}

B RILLOUIN scattering (BS) is a optical process resulting from the interaction between an incident lightwave and an acoustic waves in a medium and giving rise to backward propagating frequency-shifted light [1]. The mechanism of this scattering is the following: thermally excited acoustic waves generate through the elasto-optic effect a refractive index grating that propagates at the acoustic velocity in the material. This moving grate reflects the incident light and causes this backscattered light to be precisely shifted in frequency through the Doppler effect.

The Brillouin frequency shift is given by

$$
\nu_{B}=\frac{\omega_{A}}{2 \pi}=\frac{2 n V_{A}}{\lambda_{P}}
$$

where $\omega_{A}$ is the acoustic wave's frequency, $V_{A}$ is the acoustic wave's velocity in the optical fiber, and $n$ is the modal refractive index at the pump wavelength $\lambda_{P}$.

Stimulated Brillouin Scattering (SBS) is a nonlinear parametric process in the optical fiber coupling two contrapropagating lightwaves showing the exact frequency difference given by the Brillouin shift (1) due to Doppler effect. In this case, the acoustic wave is generated by the electrostriction resulting from the interference of the two lightwaves and plays the role of the

Manuscript received July 27, 2007; revised March 13, 2008; accepted April 15,2008 . Published July 16, 2008 (projected). Financial support from the European COST Action 299 FIDES, Spanish Comision Interministerial de Ciencia y Tecnologia within project TEC2007-67987-C02 and FEDER funds is acknowledged. This paper was presented in part at the Third European Workshop on Optical Fiber Sensors in Naples, Italy, and was published in its proceedings. The associate editor coordinating the review of this paper and approving it for publication was Dr. Brian Culshaw.

S. Diaz and M. Lopez-Amo are with the Departmento de Ingeniería Eléctrica y Electrónica, Universidad Pública de Navarra, Campus de Arrosadía s/n, 31006 Pamplona, Spain (e-mail: silvia.diaz@unavarra.es).

S. Foaleng-Mafang and L. Thévenaz are with the Laboratory of Nanophotonics and Metrology, Swiss Federal Institute of Technology, Lausanne CH-1015, Switzerland (e-mail: stella.foalengmafang@epfl.ch; Luc.Thevenaz@ epfl.ch).

Digital Object Identifier 10.1109/JSEN.2008.926963 idler wave in the parametric process. The net result of the interaction is a power transfer from the higher frequency optical wave to the lower frequency wave. This latter will, therefore, experience gain and the former loss. Within optical fibers the spectral characteristics of the Brillouin gain depend not just on the type of fiber (dopants concentration, index profile, material ...), but also on the relative polarization of the pump and probe lightwaves, and on temperature and strain applied to the fiber. This dependence on extrinsic parameters suggests the possibility of using SBS as a sensor to determine environmental variations along the whole length of the sensing fiber [2], [3].

Since the Brillouin shift essentially depends on the refractive index and on the acoustic velocity, that in turn depends on environmental quantities such as temperature or strain, its determination can be used for sensing [4], [5].

A Brillouin Optical-Fiber Time-Domain Analyzer, or BOTDA, was initially developed as an instrument enabling nondestructive evaluation of optical fiber attenuation [6], based on the Brillouin interaction between two counterpropagating lightwaves generated by two laser sources placed at the extremities of the fiber under test.

The majority of methods for BOTDA [4], [6] used two distinct lasers for generating pump and probe signals. This requires excellent frequency locking between the two lasers to secure the $1 \mathrm{MHz}$ stability for accurate measurement. Niklés et al. [5], [7]-[9] proposed a simple way to achieve an ideal stabilization of the frequency difference in a BOTDA. The configuration uses a microwave generator and a $\mathrm{LiNbO}_{3}$ electro-optic modulator (EOM) to generate pump and probe signals from one single laser source. This configuration, however, suffers from sensitivity to optical noise, as a result from the bidirectional propagation of optical waves showing the same frequency along the optical fiber.

In this paper, a novel configuration that avoids as much as possible the bidirectional propagation of waves showing the same optical frequency is presented [10]. This drastically reduces the optical noise resulting from the superposition of coherent waves with the same frequency, but showing a randomly fluctuating phase difference as generated from spurious reflections and Rayleigh scattering in the system. The random phase difference generates an important intensity noise and is frequently observed in bidirectional fiber optics systems.

\section{EXPERIMENTAL SETUP}

The block diagram of the setup is shown in Fig. 1. The laser light is first split into probe and pump channels. The laser used was a standard single frequency DFB laser diode at a wavelength of $1557 \mathrm{~nm}$ and with a $30 \mathrm{~mW}$ output power. The light in the probe channel is first modulated by an EOM that is a 


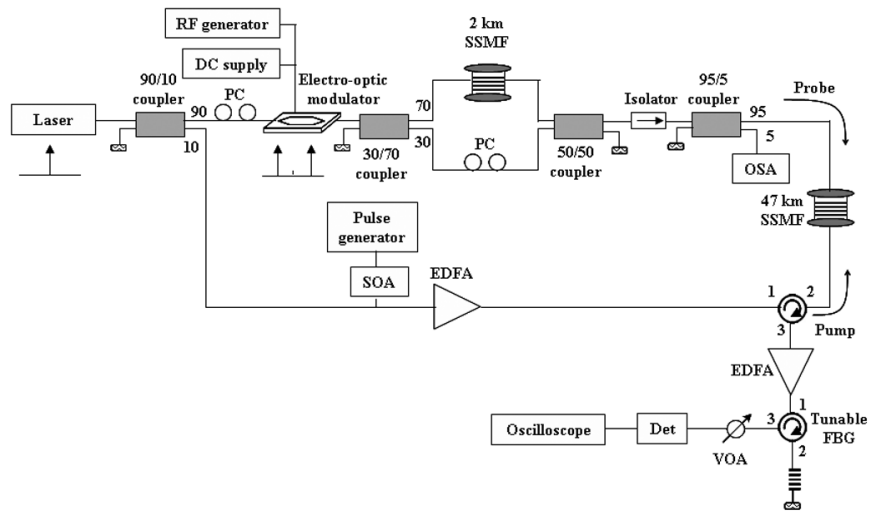

Fig. 1. Novel low-noise configuration for distributed Brillouin gain spectrum measurement.

guided-wave Mach-Zehnder $\mathrm{LiNbO}_{3}$ integrated device based on the proton exchange technology, showing a modulation bandwidth of $12 \mathrm{GHz}$ and a $30 \mathrm{~dB}$ extinction ratio. This is a key element of the setup since it is used for the generation and frequency tuning of the probe signal. The frequency shift on the laser light is achieved by simply applying a $\mathrm{CW}$ microwave signal in the frequency range of the Brillouin shift on the EOM electrodes. This creates sidebands in the laser spectrum. This technique makes the control of the probe optical frequency very convenient and reliable by adjusting the microwave modulation signal frequency. The DC bias setting on the EOM just determines the amount of transmitted power at the fundamental frequency. The DC bias condition on the EOM electrodes is set, so that the optical power at the laser original wavelength is kept as small as possible. This minimal power amount depends on the EOM characteristic extinction ratio that is specially selected to exceed $35 \mathrm{~dB}$.

The other fiber channel is gated by a SOA, model Qphotonics QSOA-1550, with $40 \mathrm{~nm}$ spectral width at a central wavelength of $1534 \mathrm{~nm}$ and $2 \mathrm{~mW}$ output power, operated in a gain-switched mode to properly shape the pump pulse with a high on-off ratio $(45 \mathrm{~dB})$. This gives the system an inherent stability, as far as frequency drifts of the laser are concerned. The pump pulse provides gain to the probe signal during its forward propagation through the stimulated Brillouin scattering process, while the $\mathrm{CW}$ probe is amplified on the way back.

To avoid any gain fading effect resulting from the polarization-dependent Brillouin interaction, we have built and developed a new passive polarization scrambler. It consisted of two branches, one with a $2 \mathrm{~km} \mathrm{SSMF}$ and another with a polarization controller, and two couplers, resulting in a highly unbalanced Mach-Zehnder interferometer. The arm length difference is much larger than the laser coherence length, so that they combine incoherently in the output coupler. The states of polarization in the two arms are set orthogonal before the recombination, so that the phase noise results in a perfect and passive polarization scrambling without intensity noise. The couplers' splitting ratios were adjusted to compensate the loss in the delaying fiber, so that the same amount of power is obtained from the two arms after the recombination to secure a uniform scrambling.
It was placed after the EOM and before the isolator, from which the light is no longer subject to polarization dependent elements. It must be pointed out that this kind of passive scrambling is absolutely perfect, but is restricted to $\mathrm{CW}$ waves since the delay present in one arm of the scrambler would entirely modify the modulation content. It is robust, cost-effective, and does not require any power supply. In the other channel, the pump pulse is generated by a SOA, in which the gain is gated using a train of electric pulses. The SOA shows an ON-OFF ratio over $40 \mathrm{~dB}$, so that the presence of light at the original laser frequency is extremely low when the SOA is in the OFF state, i.e., when the pump pulse is off. In addition, there is absolutely no light generated at the sidebands frequencies. Hence, the configuration secures a total absence of intensity noise at the sideband frequencies that were the main source of noise in the former configuration. The output is then boosted by an EDFA.

The signals from each channel are then directed to each end of the sensing fiber. There is conceptually no wave showing the same frequency propagating in the two opposite directions through the fiber, except the small residual light at the original laser frequency that results from the finite extinction ratio of the EOM, which can interfere with the Rayleigh light from the intense pump pulse.

Light from the probe channel is extracted at the fiber output using an optical circulator. This signal is leveled up using another EDFA and then filtered using a very narrowband fiber Bragg grating filter $(<0.1 \mathrm{~nm})$ to transmit only one sideband onto the detector. This filtering is crucial: it eliminates the unwanted modulator sideband that reduces the measurement contrast and any presence of the pump frequency due to the finite extinction ratio of the EOM that would generate substantial optical noise at the detection when combined with the Rayleigh light from the pump pulse.

When the modulation frequency $f_{m}$ is close to the Brillouin frequency shift $v_{B}$, the first lower sideband lies in the Brillouin gain spectrum generated by the pump and is amplified through the Brillouin interaction. As a matter of fact, the Brillouin gain spectrum can be determined by simply sweeping the modulation frequency $f_{m}$, and recording the probe intensity. The frequency spacing between the first upper and first lower sidebands is twice the frequency modulation $f_{m}$, (approximately $22 \mathrm{GHz}$ ), and corresponds to a wavelength separation of $0.25 \mathrm{~nm}$ at $1.55 \mu \mathrm{m}$. As the first upper sideband is not relevant for the measurement and even has a negative effect on the contrast, it is filtered out just before acquisition using a tunable fiber Bragg grating of $0.1 \mathrm{~nm}$ bandwidth.

\section{RESULTS}

The main performances of our Brillouin optical fiber system may be summarized as follows.

- The massive noise reduction by more than $15 \mathrm{~dB}$ down to optical shot noise has made a $50 \mathrm{~km}$ sensing range possible without any amplification along the sensing fiber to restore the pump power.

- The ultimate spatial resolution of the Brillouin sensor depends on the sensing range since the pump pulse must be made longer to maintain a sufficient gain after experiencing 


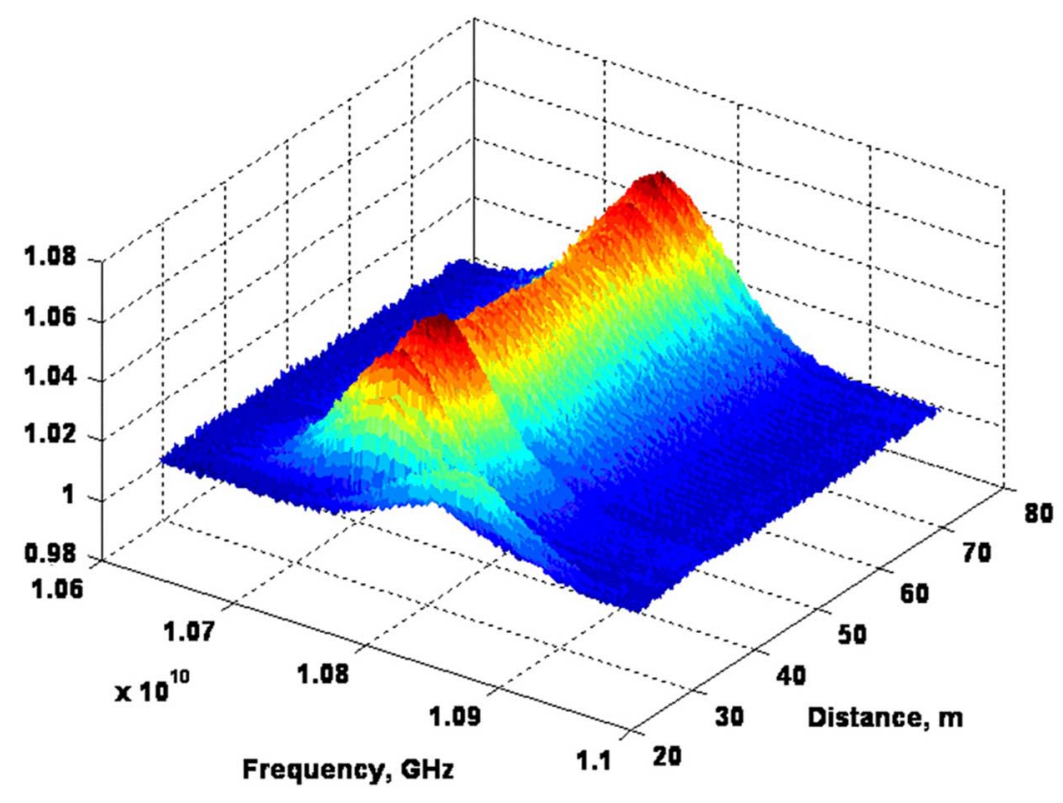

Fig. 2. Generation of a SD-surface representing the raw Information obtained from the gain traces relative to all the different frequencies during trw spectral scan. The quality of the traces, obtained after a $16 \mathrm{X}$ averaging, illustrates the massive reduction of noise.

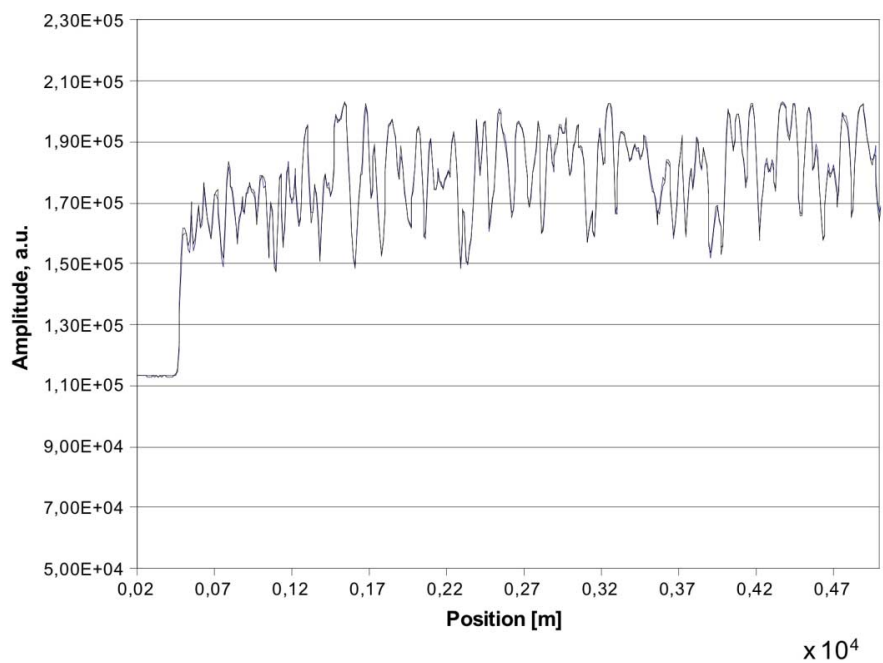

Fig. 3. Trace of maximum Brillouin gain over $5 \mathrm{~km}$ of the SSMF fiber without polarization control.

the propagation loss through the fiber. The actual best spatial resolution amounts roughly to $2 \mathrm{~m}$ for $40 \mathrm{~km}$-range and $7 \mathrm{~m}$ for $50 \mathrm{~km}$. For ranges shorter than $5 \mathrm{~km}$, a $30 \mathrm{~cm}$ resolution was obtained.

- For short ranges $(<5 \mathrm{~km})$, a $16 \mathrm{X}$ averaging results in perfect measurements. For very long ranges, 256X averaging is required.

- A new passive polarization scrambling method was successfully implemented, resulting in a perfectly flat amplification profile along the fiber and reducing polarization fading to zero.

The overall information carried by the frequency-distance distribution of the Brillouin amplification is obtained by performing a frequency sweep of the probe lightwave.

The data acquisition system was controlled using a LabView program and it acquires a normalized amplification trace at

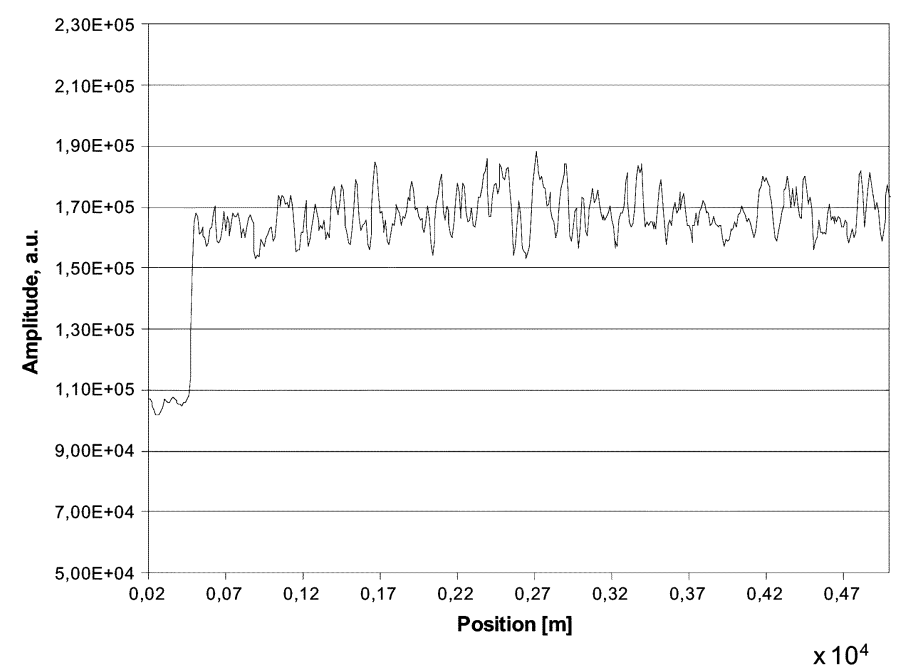

Fig. 4. Trace of maximum Brillouin gain over $5 \mathrm{~km}$ of the SSMF fiber with polarization control.

each frequency step, measuring the local amplification of the probe signal, while crossing the counterpropagating pump pulse. A simple time-of-flight to in-fiber position conversion is performed knowing the speed of propagation $\nu_{\mathrm{g}}$ of the light pulse launched into the fiber.

The trace is acquired by a $125 \mathrm{MHz}$ photodetector and an oscilloscope acquisition PCI-board working at $2 \times 10^{9}$ samples/s.

The scan in the time/distance and frequency domain can be viewed as a 3D-surface (Fig. 2) representing the spectral distribution of the Brillouin gain at any location along the fiber.

The results obtained with and without the polarization scrambler are compared (Figs. 3 and 4). As stated before, this part of the setup behaved as a Mach-Zehnder interferometer. As shown in Fig. 4, the results are greatly improved in the case of using the polarization scrambler. 


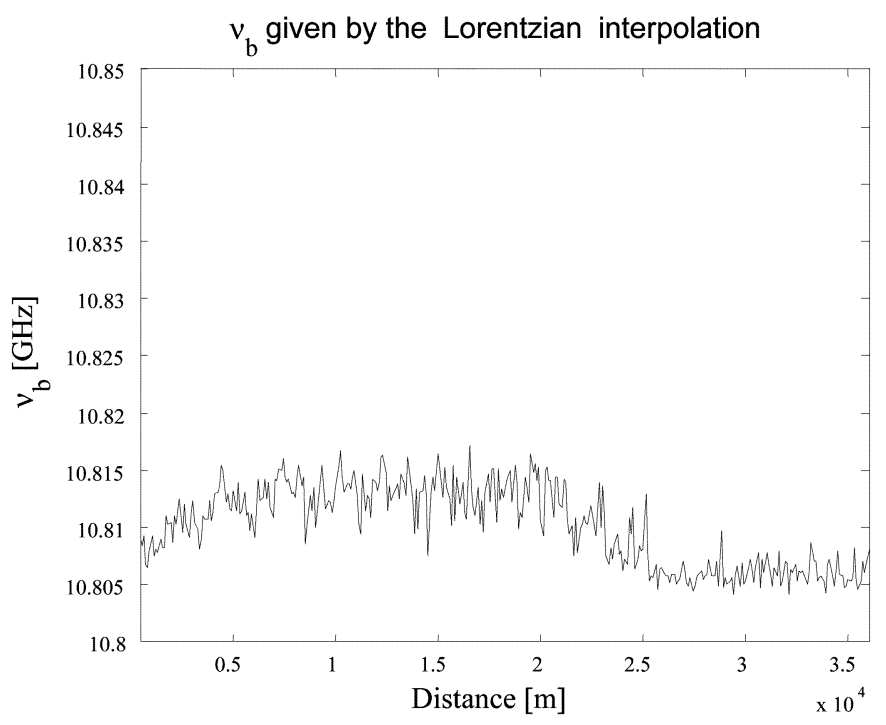

Fig. 5. Trace of maximum Brillouin gain along the first kms of the SSMF fiber without polarization control.

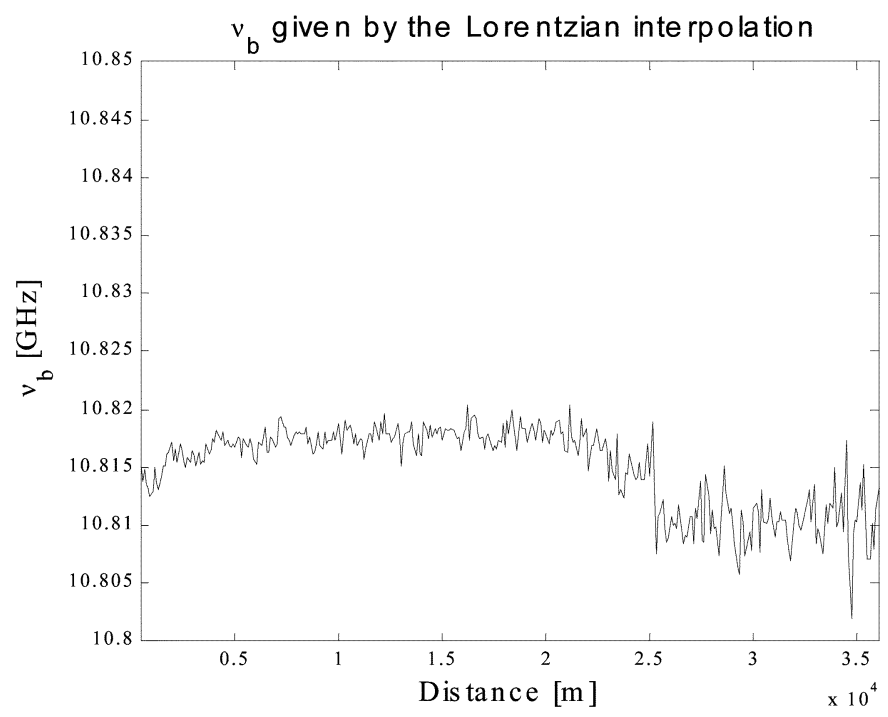

Fig. 6. Trace of maximum Brillouin gain along the first kms of the SSMF fiber with polarization control.

The results of Fig. 4 were obtained using 256 averages in the oscilloscope. Shown is the $47 \mathrm{~km}$ of SSMF. The graph is nearly flat in the case of using the polarization scrambler system, as represented in Fig. 4. The mean Brillouin frequency is $10.81 \mathrm{MHz}$ in both cases.

The resultant maximum Brillouin gain is depicted in Figs. 5 and 6 and when there was not included the passive polarization scrambler and in the case of using the polarization scrambler, respectively. A clear improvement is observed on the first kilometers, that fades out for large distances. This is certainly due to the loss penalty on the signal due to the polarization scrambler.

As before, there is a clear improvement when the polarization control given by the Mach-Zehnder interferometer was implemented. A $33 \mathrm{kHz}$ maximum variation in the Brillouin frequency was observed in the case of using the polarization scrambler, whereas the change was increased to more than $50 \mathrm{kHz}$, as shown in Fig. 5, when there is no polarization control.

\section{CONCLUSION}

A novel BOTDA configuration able to perform a distributed measurement of the Brillouin gain spectrum using a single laser source has been developed. This configuration is designed to minimize all sources of intensity noise, resulting in extreme performances in terms of range, spatial resolution, and acquisition time. A spatial resolution of $7 \mathrm{~m}$ over a $47-\mathrm{km}$ fiber length has been obtained. Actually, the range is limited by the modulation instability that actively spreads the spectrum of the pump pulse and longer fiber lengths can certainly be measured using a fiber showing a normal group velocity dispersion. A new passive polarization scrambler made of an unbalanced Mach-Zehnder interferometer was implemented and made possible the measurement of Brillouin frequencies with a maximum variation of $33 \mathrm{kHz}$ and much reduced Brillouin gain oscillations.

\section{REFERENCES}

[1] G. P. Agrawal, Non linear Fiber Optics, 3rd ed. San Diego, CA: Academic, 2001.

[2] L. Thévenaz, M. Niklés, A. Fellay, M. Facchini, and P. Robert, "Applications of distributed Brillouin fiber sensing," in SPIE Proc. 3407 Int. Conf. Appl. Opt. Metrology, P. K. Rastogi and F. Gyimesi, Eds., Balatonfüred, Hungary, 1998, pp. 374-381.

[3] L. Thévenaz, M. Facchini, A. Fellay, M. Niklés, and P. Robert, "Evaluation of local birefringence along fibres using Brillouin analysis," in in Proc. 4th Opt. Fibre Measure. Conf., 1997, pp. 82-85.

[4] X. Bao, J. Dhliwayo, N. Heron, D. J. Webb, and D. A. Jackson, "Experimental and theoretical studies on a distributed temperature sensor bases on Brillouin scattering," IEEE J. Lightw. Technol., vol. 13, no. 7, pp. 1340-1348, July 1995.

[5] M. Niklés, L. Thévenaz, and P. A. Robert, "Brillouin gain spectrum characterization in single-mode optical fibers," IEEE J. Lightw. Technol., vol. 15, pp. 1842-1851, 1997.

[6] T. Horigushi and M. Tateda, "Optical- fiber-attenuation investigation using stimulated Brillouin scattering between a pulse and a continuous wave," Opt. Lett., vol. 14, no. 4, pp. 408-410, Apr. 1989.

[7] L. Thévenaz, S. Le Floch, D. Alasia, and J. Troger, "Novel schemes for optical signal generation using laser injection locking with application to Brillouin sensing," Measure. Sci. Technol., vol. 15, pp. 1519-1524, Aug. 2004.

[8] M. Niklés, L. Thévenaz, and P. Robert, "Measurement of the distributed Brillouin-gain spectrum in optical fibres by using a single laser source," in Proc. Int. Conf. Opt. Fiber Commun., San Jose, CA, 1994, pp. 89-90, paper WF1.

[9] M. Niklés, L. Thévenaz, and P. Robert, "Simple distributed fibre sensor based on Brillouin gain spectrum analysis," Opt. Lett., vol. 21, pp. 758-760, May 1996.

[10] S. Diaz, S. F. Mafang, M. Lopez-Amo, and L. Thévenaz, "High performance Brillouin distributed fibre sensor," in Proc. Eur. Workshop on Opt. Fibre Sensors, Naples, 2007.

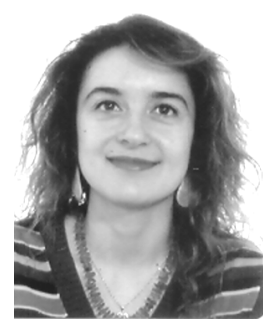

Silvia Diaz was born in Valencia, Spain. She received the Telecommunications Engineer degree and the Ph.D. degree from the Universidad Pública de Navarra, Pamplona, Spain in 2002 and 2007, respectively. In October 2003, she was a visiting $\mathrm{Ph}$.D. student at the Photonics Group in Universidad de Cantabria, Spain, working on signal processing with distributed fiber-optic networks.

In November 2004, she became an Assistant Professor in the Department of Electrical and Electronical Engineering, Universidad Pública de Navarra. During the summer of 2006, she was a visiting Ph.D. student at the NAM Laboratory at the École Polytéchnique Fédérale of Lausanne, Switzerland, working on Brillouin Scattering. Her research interests are in distributed nonlinear amplified networks for optical fiber sensors, Raman amplifiers, erbium-doped amplifiers, and their applications in wavelength-division-multiplexing communication systems and networks. 


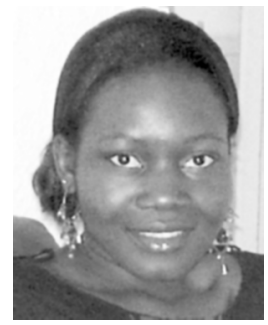

Stella Foaleng Mafang was born in Akonolinga, Cameroon. She received the degree of Dottoressa in Ingegneria de l'informazione from the Universitá degli studi di Perugia, (UNIPG), Italy, in 2003, the M.S. degree in electrical and electronic engineering from the Swiss Federal Institute of Technology of Lausanne (EPFL), Switzerland, in 2006. She is currently working towards the Ph.D. degree at the Swiss Federal Institute of Technology of Lausanne (EPFL).

Her research interests include, distributed fiber optical sensing, optical information storage based on Stimulated Brillouin scattering.

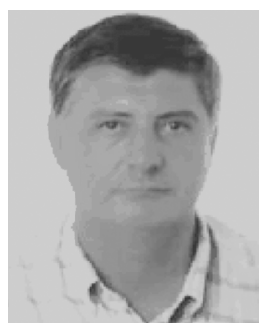

Manuel Lopez-Amo (M'91-SM'98) was born in Madrid, Spain, in June 1960. He received the Telecommunications Engineer degree and the Ph.D. degree from the Universidad Politécnica de Madrid, Spain, in 1985 and 1989, respectively. He was the recipient of the extraordinary doctorate prize of the Universidad Politécnica de Madrid.

From 1985 to 1989, he was a Lecturer in Optical Communications and Electronics at the Photonic Technology Department, Universidad Politécnica de Madrid. During 1987, he was a visiting Ph.D. student at INTEC of the University of Ghent, Belgium, working on integrated optics. In January 1990, he became an Associate Professor at the Photonic Technology Department, Universidad Politécnica de Madrid. In 1992, he was a Visiting Researcher at British Telecommunication Research Laboratories, Ipswich, U.K., working in amplified fiber-optic networks. In 1996, he moved to the Universidad Publica de Navarra, Pamplona, Spain, where he became a
Full Professor in the Electrical and Electronical Engineering Department and is currently the Head of the Optical Communications Group of this department. He was also the current Chairman of the Optoelectronic Committee of Spain. $\mathrm{He}$ has been the Head of the Electrical and Electronic Engineering Department and the Engineering Faculty of this university. He has been the leader of more than 20 research projects and he has coauthored more than 100 works in international refereed journals and conferences related with fiber-optic networks, fiber-optic components, optical amplifiers, fiber-optic processors, fiber-optic sensors, and integrated optics.

Prof. Lopez-Amo is a member of the technical committees of the International Conference on Fiber-Optic Sensors (OFSs), the European Workshop on Optical Fiber Sensors (EWOFS), and European COST 299 Action, among others.

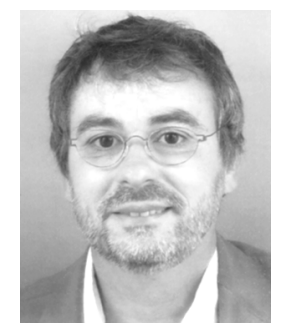

Luc Thévenaz received the M.Sc. degree in astrophysics from the Observatory of Geneva, Geneva, Switzerland, in 1982 and the Ph.D. degree in physics from the University of Geneva in 1988.

In 1988, he joined the Swiss Federal Institute of Technology of Lausanne (EPFL), where he currently leads a research group involved in photonics, namely, fiber optics and optical sensing. Research topics include Brillouin-scattering fiber sensors, slow and fast light, nonlinear fiber optics and laser spectroscopy of gases. During his career, he stayed at the PUC University, Rio de Janeiro, Brazil, at Stanford University, at the Korea Advanced Institute of Science and Technology (KAIST), and at Tel-Aviv University. In 2000, he co-founded the company Omnisens that is developing and commercializing advanced photonic instrumentation.

Dr. Thévenaz is Chairman of the European COST Action 299 "FIDES: Optical Fibers for New Challenges Facing the Information Society." 